Reptilase and Thrombin Clotting time of Plasma from Patients Treated with Streptokinase

z. S. LATAllo AND W. MALANOWICZ (Institute of Nuclear Research and Institute of Cardiology, Warsaw)

Increasing clinical experience has gradually diminished the number of laboratory tests required for monitoring thrombolytic therapy. Still wider and wider use of plasminogen activators, often without a specialized laboratory basis, makes it imperative that some easily performed and reproducible tests should be at our disposal. Such conditions seem to be fulfilled by a simple assay of plasma clotting time. Usually thrombin was used as the clotting agent for this purpose. The test could not, however, be applied or would give erroneous results if heparin had been given simultaneously or following treatment with thrombolytic agents. The use of reptilase instead of thrombin is therefore proposed.

In contrast to the observations made by some others, our previous studies (Latallo and Teisseyre, 1971) indicated that the addition of fibrinogen degradation products (FDP) to normal plasma resulted in parallel prolongation of both the thrombin and the reptilase clotting times. A comparison of both types of clotting tests was made on plasma samples from four patients obtained at various stages of treatment with Kabikinase (Kabi, Stockholm). Plasma was obtained from blood taken into a mixture of EACA and sodium citrate with the following assay system: $0.2 \mathrm{ml}$ of plasma and 0.1 $\mathrm{ml}$ of enzyme, $37^{\circ}, \mathrm{pH}=7 \cdot 8$. Thrombin was dissolved in the same carrier solution as reptilase to a concentration which gave an identical clotting time of noimal plasma for both enzymes. Standardized preparation Reptilase R (Pentapharm, Basel) was used.

Very good agreement was observed in values for clotting times produced by either enzyme. Both equally depend upon the concentration of fibrinogen and/or FDP. Comparison of clotting times of undiluted plasma samples and on samples mixed in a 1:1 ratio with normal plasma allowed workers to distinguish whether the observed prolongation was due to a very low content of fibrinogen or to a high concentration of fibrinogen degradation products.

\section{Preliminary Results of the Reptilase Clotting Time and Protamine Test in Patients Undergoing Open Heart Surgery with Extracorporeal Circulation}

M. PALESTER-CHLEBOWCZYK, E. STRZYZEWSKA, AND Z. S. LATALlo (Institute of Tuberculosis and Institute of Nuclear Research, Warsaw)

Extracorporeal circulation is usually accompanied by a marked decrease in fibrinogen level of plasma. This may be due simply to dilution of blood in the apparatus but also to the activation of intravascular coagulation and/or fibrinolysis. The changes may occur quite suddenly and appropriate therapeutic measures have to be undertaken as soon as possible.

The protamine test and reptilase clotting time have been already proposed for the detection and evaluation of the intravascular formation of fibrin and fibrinogen degradation products respectively (Latallo, Wegrzynowicz, Teisseyre, and Kopec, 1971). Previous work (Palester-Chlebowczyk, Strzyzewska, Sitkowski, Olender, Wegrzynowicz, and Latallo, 1971) ${ }^{1}$ indicated that the protamine test, if run under proper conditions (at $37^{\circ}, 0 \cdot 1-0.2 \%$ final concentration of protamine sulphate, $p \mathrm{H}$ around 7.8), may serve as a rapid method for detection of intravascular fibrin formation. Further results confirm these observations.

Since heparin given during surgery seriously interferes with many clotting tests, the reptilase clotting time (which is not affected by the presence of the drug) seemed to be particularly convenient for detection of active products of fibrinogen proteolysis (Latallo and Teisseyre, 1971).

Clotting time measurements were performed at $37^{\circ}, p \mathrm{H}=7 \cdot 8$ in a system: $0.2 \mathrm{ml}$ of citrated plasma and $0.1 \mathrm{ml}$ of standardized preparation of reptilase (Reptilase R, Pentapharm, Basel). The test was repeated at short intervals before, during, and after surgery, and the results were compared with assays of the euglobulin lysis time and fibrinogen content in plasma.

In all cases in which the euglobulin lysis time was significantly shortened, the clotting time was prolonged. Some prolongation was observed also in samples with prolonged euglobulin lysis time. This might indicate that the fibrinogen degradation products remain in circulation for some time after the activation of the fibrinolytic system.

It is concluded that the two tests described here might be conveniently applied as simple screening tests during open heart surgery.

Methodology for the Control of Thrombolytic Therapy NORMA ALKJAERSIG (Washington University School of Medicine, St Louis, Missouri)

Methods required for the control of thrombolytic therapy include (1) methods for measuring the intensity of the induced plasma thrombolytic state, (2) methods for assay of other plasminogen-plasmin system components altered by therapy, (3) methods for determining the secondary effects of the treatment

${ }^{1}$ Scand. J. Haemat., 1971 (In press). 
on the blood coagulation system, and (4) finally, a new class of methods recently introduced by our laboratory for determining the presence in vivo of thrombotic/blood hypercoagulable states.

The accurate measurement of induced plasma thrombolytic activity is crucial to the rational use of thrombolytic agents. The usual methods for measuring induced plasma thrombolytic activity are, because of their simplicity, the euglobulin clot lysis method, or the application of the patient's euglobulin to a fibrin plate. While these methods are relatively sensitive and can be used as a measure of low-grade fibrinolytic activity, they are not well suited to the control of thrombolytic therapy because substrate concentrations (plasminogen and fibrinogen) change during the infusion period and also because in precipitating the euglobulin fraction, the majority of the plasmin inhibitors are left in the supernatant.

The method of choice for determining plasma thrombolytic activity in patients undergoing thrombolytic therapy is the ${ }^{125} \mathrm{I}$ tagged clot assay (Fletcher, 1964; Alkjaersig, Fletcher, and Sherry, 1959), in which the patient's plasma is incubated for a standard time period with a standard plasma small clot, incorporating ${ }^{125}$ I tagged fibrin and a amount of acid-soluble plasminogen. Following incubation, the mixture is centrifuged and plasma thrombolytic activity is calculated from assay for supernatant radioactivity. This radiochemical method can be modified so that it is sufficiently sensitive to measure the plasma thrombolytic activity from normal resting man. When used to monitor urokinase therapy, there is a high degree of correlation between dose and circulating thrombolytic activity compared to data obtained by the use of the patient's plasma on unheated fibrin plates. The unheated fibrin plate method, though satisfactory, is far too slow for the control of therapy, 을 for the data are obtained only 18-24 hours after obtaining the sample while data from the ${ }^{125}$ I tagged $\stackrel{\vec{\rho}}{\overrightarrow{3}}$ clot assay is available some 90 minutes after receiving음 the sample. Though we introduced these radio- 들 chemical methods some years ago, they have never $\frac{\bar{s}}{\frac{\sigma}{\sigma}}$ been particularly popular because the preliminary ${ }_{0}$ step of iodinating fibrinogen was regarded as difficult. With the increasing commercial availability of iodinated fibrinogen preparation;, methodological. difficulties may be substantially reduced and these $\vec{\omega}$ valuable methods may receive wider use.

Recently, we introduced a new method, plasma fibrinogen chromatography, for the detection and is quantification of specific fibrinogen/fibrin com- $v$ plexes and derivatives, characteristic of in vivo thrombosis, blood hypercoagulability, intravascular $N$ coagulation, and fibrinolysis (Fletcher, Alkjaersig, o O'Brien, and Tulevski, 1970). Such assays, always abnormal, that is, showing gross blood hypercoagula- bility/thrombosis patterns, in patients requiring $气$ thrombolytic therapy, are characteristically altered $<$ when effective thrombolytic therapy is administered. $\overrightarrow{0}$ This alteration in the pattern, from gross blood N hypercoagulability/thrombotic-type pattern to that of fibrinogen first derivative, indicates a change in blood rheological properties. Insofar as a limited experience with urokinase therapy in acute cerebrovascular syndromes is relevant, it appears that $\Phi$ this alteration in the plasma fibrinogen chromatogram may be a most novel and useful indicator of satisfactory dosage of thrombolytic agents. At least in cerebrovascular disease, it appears that we can use smaller dosage than has hitherto been suggested, a step both greatly reducing the hazards of the treatment and also resulting in substantial saving of a very expensive drug. 